\title{
Estado nutricional y horas de sueño en escolares de enseñanza básica de la ciudad de Punta Arenas, año 2016
}

\section{Nutritional status and hours of sleep among elementary school students in the city of Punta Arenas in 2016}

\section{ABSTRACT}

The aim of the study was to study the nutritional status and the average number of sleep hours on weekdays and weekend among 6 to 15-year-old schoolchildren in the city of Punta Arenas, Chile. Subjects and methods: A simple random sample of 481 schoolchildren from two schools (one state-subsidized and one public) was obtained. To evaluate nutritional status, we measured weight, height and triceps skinfold. In order to determine usual sleep duration, the Spanish version of the Pediatric Sleep Questionnaire was used and the results were compared with the recommendation given by the National Sleep Foundation. Results: $60.4 \%$ of schoolchildren showed malnutrition due to excess, slept on average $8.70 \pm 1.07$ hours on weekdays and $9.85 \pm 1.55$ hours on weekends. Conclusion: Obese children had less compliance with recommendations, however differences were not statistically significant.

Key words: Obesity, schoolchildren, nutritional status, sleep hours.

\section{INTRODUCCIÓN}

La ubicación geográfica extrema de la región de Magallanes, condiciona a sus habitantes a noches muy cortas o muy largas según la época estacional, afectando la duración habitual del sueño de sus habitantes, con días que pueden alcanzar las 17 horas, mientras que en invierno esta condición se ve disminuida a sólo 7 horas y 30 minutos ${ }^{1}$, exponiendo a la población a una gran cantidad de horas de luz artificial, factor que podría condicionar, la alta tasa de malnutrición por exceso tanto en adultos como en niños. Datos recientes, muestran que la prevalencia de obesidad en la región en pre kínder corresponde a un $26,4 \%$, kínder $27,3 \%$ y un $29,0 \%$ en primer año básico, superando el promedio nacional ${ }^{2}$.

La disminución de las horas de sueño es una realidad mundial. En la década de 1960, la duración media del sueño en los Estados Unidos fue más de 8 horas, pero cayó a 7 horas en 1995. En el año 2005, una encuesta realizada en
Paola Aravena, Andrea Mansilla, Andrea Pangue, Vanesa Needham, Carmen Muñoz.

Carrera de Nutrición y Dietética. Departamento de Kinesiología, Facultad de Ciencias de la Salud, Universidad de Magallanes, Punta Arenas, Chile.

Dirigir la correspondencia a: Profesor Paola Aravena Martinovic. Avenida Bulnes 01855 Casilla 113-D Punta Arenas. Chile Teléfono: 56-61-2299686 E-mail:paola.aravena@umag.cl

Este trabajo fue recibido el 30 de marzo de 2017. Aceptado con modificaciones: 16 de mayo de 2017. Aceptado para ser publicado: 28 de junio de 2017.

ese país mostró que la duración media del sueño fue de 8,4 horas en niños de 11-12 años y menos de 7 horas en adolescentes de 16-17 años. Informes recientes de China, Australia y otros países, también indicaron una disminución de la duración del sueño entre los niños de 6 a 18 años, considerándose la corta duración del sueño como un fenómeno global ${ }^{3}$.

La evidencia científica señala que los durmientes cortos y el aumento de la exposición a la luz artificial durante la noche son paralelo al aumento global de la prevalencia de la obesidad y los trastornos metabólicos ${ }^{4}$.

Un estudio desarrollado en el Reino Unido y aplicado en niños y adolescentes, comparó que aquellos que duermen menos de 10,5 horas en la noche a la edad de 30 meses, presentaban un $45 \%$ más de riesgo de desarrollar obesidad a la edad de 7 años que aquellos que durmieron mayor cantidad de horas (> 12 horas/noche). Los niños que dormían menos de 9 horas por noche tuvieron un mayor riesgo de 
sobrepeso y obesidad $(\mathrm{OR}=1,29)$ y obesidad abdominal $(\mathrm{OR}=1,38)^{5}$.

Esta condición se debería principalmente, a que la exposición a la luz artificial (dim ligth) induce a un desajuste circadiano, desarrollando alteraciones metabólicas, simulando un síndrome metabólico y de resistencia insulínica.

Considerando que el sueño es un importante modulador del crecimiento, la maduración y la salud de los niños y adolescentes y que el buen dormir está estrechamente vinculado con la edad y el neurodesarrollo, la falta de sueño en los niños a diferencia de lo que sucede en los adultos, tendrá un impacto negativo en el desarrollo infantil, referido al comportamiento, al rendimiento académico y al crecimiento. Sin duda que también afectará la dinámica y la calidad de vida familia ${ }^{6}$.

El objetivo del estudio fue conocer el estado nutricional y la duración habitual del sueño y analizar cuál es la contribución del sueño a la elevada prevalencia de obesidad en los escolares chilenos de 6 a 15 años de edad de la ciudad de Punta Arenas.

\section{MATERIAL Y METODOS}

Investigación de enfoque cuantitativo, de tipo descriptiva correlacional.

\section{Sujetos y Métodos}

La muestra estuvo constituida por 481 individuos de ambos sexos, entre 6 y 15 años de edad, quienes cursaban entre $1^{\circ}$ a $8^{\circ}$ año básico en dos establecimientos educacionales de la ciudad de Punta Arenas, entre el año 2016. La técnica de muestreo utilizada fue la fórmula aleatoria simple. Se calculó considerando un 95\% de confianza y un error muestral del $5 \%$.

\section{Criterios de Inclusión}

Entre los criterios de inclusión estaba ser escolar entre 6 y 15 años de edad, de uno de los 2 establecimientos estudiados y los criterios de exclusión correspondieron a aquellos escolares que no completaron la evaluación antropométrica o la encuesta y/o que presentaron trastornos del sueño en tratamiento médico.

Las variables antropométricas estudiadas, previa aprobación del consentimiento informado a padres $y / o$ apoderados fueron: peso, talla y pliegue tricipital. Las mediciones antropométricas fueron ejecutadas por estudiantes de $3^{\circ}$ año de la carrera de Nutrición y Dietética, de la Universidad de Magallanes.

Para determinar el peso corporal $(\mathrm{kg})$, se utilizó una balanza mecánica marca ADE, de precisión de 100 g, previamente calibrada, con el mínimo de ropa posible.

La estatura expresada en centímetros $(\mathrm{cm})$ se obtuvo al medir al escolar con un tallimetro incorporado a la balanza mecánica con graduación de 0,1 cm. La medición del pliegue cutáneo tricipital se realizó utilizando un Calliper marca Lange de precisión de $1 \mathrm{~mm}$ y presión de $10 \mathrm{gr} / \mathrm{mm}^{2}$ en el brazo izquierdo del cuerpo, tres veces consecutivas.
La circunferencia del brazo fue determinada con una cinta métrica flexible de $1 \mathrm{~mm}$ de precisión.

El índice de Masa Corporal (IMC) se calculó dividiendo el peso por la talla al cuadrado IMC $=$ Peso $(\mathrm{kg}) / \mathrm{Talla}^{2}(\mathrm{~m})$.

El diagnóstico nutricional se clasificó según la Norma Técnica de Evaluación del Estado Nutricional del niño de 6 a 18 años del Ministerio de Salud de Chile $(2003)^{7}$ y se consideró con estado nutricional bajo peso, a los escolares que se encontraban bajo el percentil 10 (p10); normal entre el percentil 10 y menor al percentil 85 (p10-p85); riesgo de obesidad, al escolar que se encontraba entre el percentil 85 y percentil 95 (p85-p95) y con obesidad al escolar que se encontraba en el p95 o superior.

El pliegue Cutáneo Tricipital (PCT) se clasificó según las tablas propuestas por Frisancho (1981), contenidas en el libro de Evaluación Nutricional del Crecimiento y Riesgo Cardiovascular y Metabólico ${ }^{8}$, considerando como contenido bajo de grasa corporal menor al percentil 10, normal de grasa corporal entre el percentil 10 y percentil 90 y exceso de grasa corporal igual o superior al percentil 90.

Las variables independientes estudiadas, fueron duración habitual del sueño nocturno durante la semana y fin de semana. Para la recolección de la información se utilizó la versión española del Sleep Pediatric Questionaire, la cual fue aplicada a los padres de los escolares.

Para calificar el cumplimiento de horas de sueño, se utilizó la recomendación entregada por la National Sleep Foundation $(2015)^{9}$, la cual recomienda 9-11 horas de sueño para los niños entre 6-13 años y 8-10 horas para los escolares entre 14 a 17 años.

Los datos fueron procesados en una planilla Excel Microsoft Office 97-2003. Para evaluar la correlación entre las variables, se utilizó el programa estadístico SPSS versión 22 con una correlación significativa en el nivel $p<0,05$.

\section{RESULTADOS}

La muestra estudiada estuvo compuesta por 481 escolares. La edad promedio fue de $9.7 \pm 0.9$ años. El peso promedio fue de $42,2 \pm 11,4 \mathrm{~kg}$ y la talla fue de $1,38 \pm 0,14 \mathrm{mts}$. El IMC promedio fue de $21,2 \pm 4,4 \mathrm{Kg} / \mathrm{m}^{2}$ y el promedio del pliegue cutáneo tricipital fue de $20,3 \pm 9,2 \mathrm{~mm}^{2}$. Respecto al diagnóstico nutricional, el 38,3\% se encontraba normopeso y un $60,4 \%$ presento malnutrición por exceso (Tabla 1 ).

Al calcular las horas de sueño de la muestra, el grupo de 6-9 años presentó una mayor duración habitual de sueño en comparación con el grupo de 10-15 años, con un promedio de $8,9 \pm 1,1$ horas para los días de semana $(\mathrm{p}=$ $0,004)$. Respecto al fin de semana los estudiados entre 6-9 años presentaron una mayor duración con $9,9 \pm 1,5$ horas $(p=0,293)$. El mayor porcentaje de cumplimiento de horas habitual de sueño, se presentó en el grupo de 10-15 años de edad, con un $84,1 \%$ durante la semana, existiendo diferencia significativa entre los grupos estudiados $(p=$ $0,001)$. Durante el fin de semana, los escolares entre 10 a 15 años presentaron mayor cumplimiento con un $65,9 \%$ $(p=0,84)$ (Tabla 2). 
En relación al cumplimiento de la recomendación de horas de sueño, según estado nutricional, los escolares diagnosticados con obesidad, presentan el porcentaje más bajo de cumplimiento con un $69,2 \%$ para los días de semana $(p=0,215)$. En cuanto al fin de semana los escolares normopeso, es el grupo que presenta menor porcentaje de cumplimiento con un $63,0 \%$, no existiendo diferencia significativa $(p=0,781)$ (Tabla 3 ).

Los resultados sugieren que aquellos escolares con porcentaje de grasa normal, son los que presentan menor cumplimiento de la recomendación habitual de sueño durante la semana, con un $69,0 \%$, no existiendo diferencia

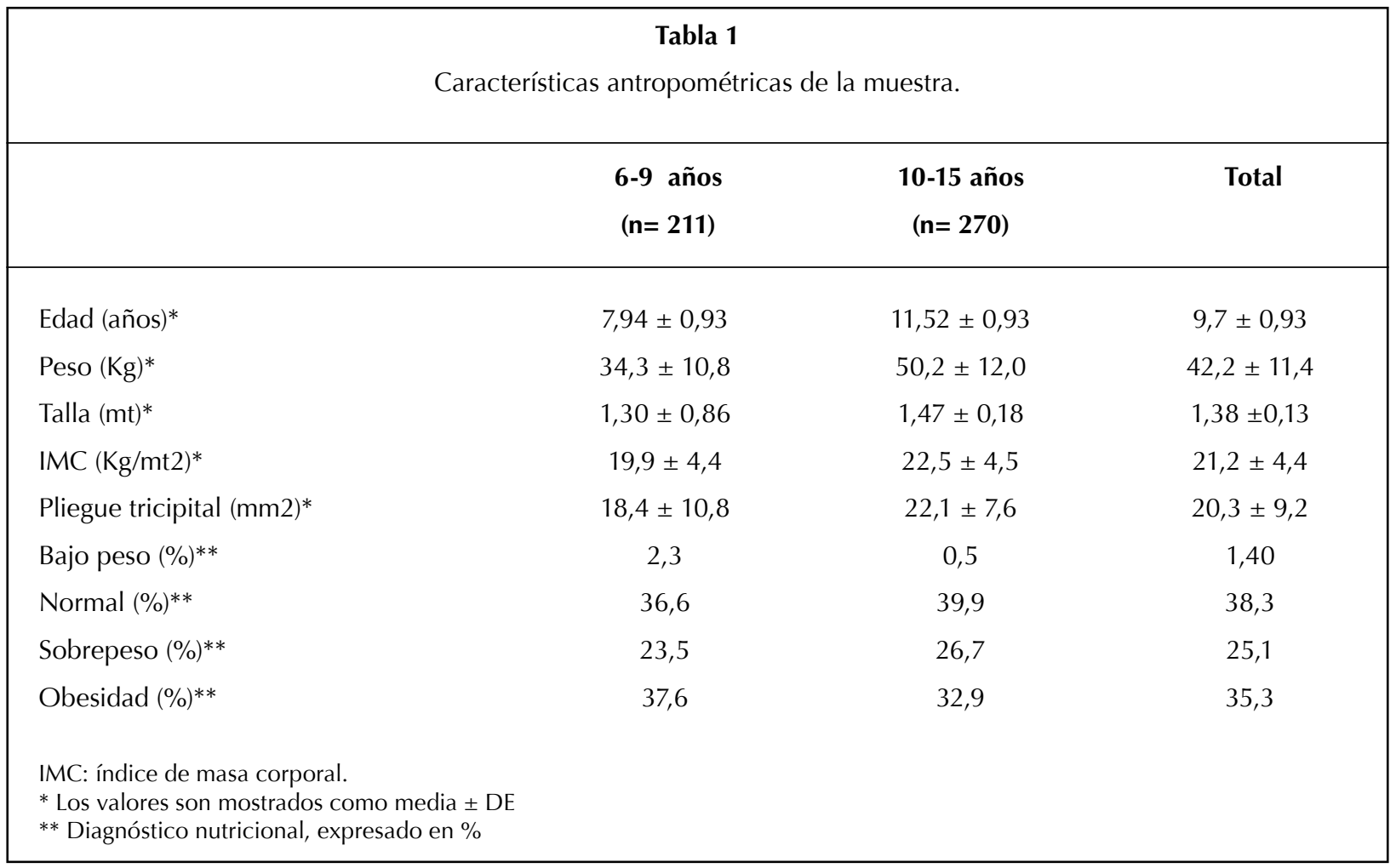

Tabla 2

Duración habitual del sueño por rango de edad.

\begin{tabular}{|c|c|c|c|c|}
\hline & $\begin{array}{c}6-9 \\
(n=211)\end{array}$ & $\begin{array}{c}10-15 \\
(n=270)\end{array}$ & $\begin{array}{c}\text { Total } \\
(n=481)\end{array}$ & $\begin{array}{c}\text { valor } \\
\mathbf{p}\end{array}$ \\
\hline Horas de sueño semana (horas)* & $8,85 \pm 1,07$ & $8,56 \pm 1,06$ & $8,70 \pm 1,07$ & 0,004 \\
\hline Horas de sueño FDS (horas)* & $9,94 \pm 1,46$ & $9,76 \pm 1,63$ & $9,85 \pm 1,55$ & 0,293 \\
\hline$\%$ de cumplimiento semana** & 58,8 & 84,1 & 71,5 & 0,000 \\
\hline$\%$ de cumplimiento FDS** & 64,9 & 65,9 & 65,5 & 0,84 \\
\hline
\end{tabular}


significativa con los otros grupos $(p=0,160)$. El menor porcentaje de cumplimiento de fin de semana se encontró en los evaluados con bajo porcentaje de grasa, corporal, con un $64,3 \%$, no existiendo diferencia significativa con los otros grupos $(p=0,983)$ (Tabla 4$)$.

\section{DISCUSION}

Los resultados encontrados en el estudio, continúan reflejando una alta prevalencia de malnutrición por exceso en la Duodécima Región, coincidiendo con los reportes entregados el año 2016 por la Junta Nacional de Auxilio Escolar y Becas (JUNAEB), los cuales indicaron que la Región de Magallanes presenta un 50,2\% de malnutrición por exceso en escolares de primer año básico ${ }^{2}$. Una de las posibles causas a este hallazgo es que en los últimos años los factores ambientales especialmente los dietarios, se han convertido en los más importantes para explicar el cambio en el estado nutricional de los escolares y en la población en general, ya que numerosos estudios muestran un incremento en el consumo de comida rápida, envasada, con alta concentración de grasa, azúcar y sal ${ }^{10}$.

Este estudio reveló que los escolares del rango de edad entre los 6-9 años presentan en promedio una mayor duración habitual de horas de sueño en comparación con los escolares de 10-15 años, existiendo diferencia significativa entre las variables. Sin embargo, al comparar los resultados obtenidos respecto al cumplimiento de la recomendación entregada por la National Sleep Foundation 2015, el mayor grado de cumplimiento de las horas de sueño se encuentra en el grupo de 10-15 años, factor que puede estar influenciado porque este rango, presenta un requerimiento menor de horas de sueño (- 1 hora) que el grupo de 6-9 años.

Por otra parte, el reporte de los padres, pudiese estar sub o sobre valorado, ya que en general los adolescentes presentan mayor autonomía e independencia en sus actividades, dentro de las cuales destacan los hábitos de utilización de dispositivos electrónicos en el dormitorio hasta altas horas de la madrugada. Este hecho es similar a lo informado en un estudio realizado en escolares de Filadelfia, en el que la disminución del tiempo de sueño estuvo asociada a actividades nocturnas, como jugar videojuegos o usar la computadora ${ }^{11}$.

Al analizar el cumplimiento de la recomendación de horas de sueño durante la semana, según estado nutricional, no se encontró diferencia significativa entre los diversos estados nutricionales, sin embargo, se observa una tendencia a disminuir las horas de sueño, al aumentar el peso corporal, resultado similar a lo encontrado en dos estudiados realizados en Chile los años 2012 y 2015, donde se indica que se encuentra una asociación entre menos horas de sueño e incremento del riesgo de sobrepeso y obesidad $^{12,13}$

Tabla 3

Cumplimiento de recomendación de sueño, según estado nutricional.

\begin{tabular}{|c|c|c|c|c|c|}
\hline & $\begin{array}{c}\text { Bajo Peso } \\
\quad(n=8)\end{array}$ & $\begin{array}{c}\text { Normal } \\
(n=184)\end{array}$ & $\begin{array}{c}\text { Sobrepeso } \\
(n=117)\end{array}$ & $\begin{array}{l}\text { Obesidad } \\
(n=172)\end{array}$ & $\begin{array}{c}\text { valor } \\
\mathbf{p}\end{array}$ \\
\hline$\%$ de cumplimiento semana*/** & 100 & 75,0 & 73,5 & 69,2 & 0,215 \\
\hline$\%$ de cumplimiento FDS*/** & 75,0 & 63,0 & 68,4 & 65,7 & 0,781 \\
\hline
\end{tabular}

\section{Tabla 4}

Cumplimiento recomendación de sueño, según \% de grasa corporal.

\begin{tabular}{|lcccc|}
\hline & $\begin{array}{c}\text { Bajo } \\
(\mathbf{n = 1 4})\end{array}$ & $\begin{array}{c}\text { Normal } \\
(\mathbf{n = 1 8 4})\end{array}$ & $\begin{array}{c}\text { Exceso } \\
(\mathbf{n = 1 1 7})\end{array}$ & $\mathbf{p}$ \\
\hline $\begin{array}{l}\text { \% de cumplimiento semana*/** } \\
\text { \% de cumplimiento FDS*/** }\end{array}$ & 100 & 69 & 75,7 & 0,160 \\
$\begin{array}{l}\text { * Para la estimación del valor p de las variables cumplimiento de recomendación de horas de sueño, se utilizó la prueba Chi². } \\
\text { ** \% de cumplimiento, calculado en base a la recomendación de sueño de la National Sleep Foundation 2015. }\end{array}$ \\
\hline
\end{tabular}


Respecto al fin de semana, no existe significancia entre los diversos grupos, evidenciándose una disminución de los porcentajes en comparación con los días de semana en todos los estados nutricionales.

Si se considera la relación obesidad/sueño, CarrilloLarco et $\mathrm{al}^{14}$ demostraron que la obesidad era un $64,0 \%$ más frecuente entre los niños con sueño de corta duración, lo que explicaría lo anteriormente descrito. Una revisión sistemática de 10 estudios realizados entre el año 2002 y 2014 reportaron una asociación positiva entre la corta duración del sueño y el riesgo de padecer obesidad, en niños en edad escolar, encontrándose un aumento de riesgo de padecer obesidad, de hasta 3 veces mayor en niños que duermen menos de 9 horas/ noche ${ }^{15}$.

Además, la restricción de sueño puede influir en el desarrollo de la obesidad a través de diversas vías biológicas, incluyendo un mayor tono simpático, aumento de la concentración plasmática de cortisol y grelina, disminución de la leptina y hormona del crecimiento, alteración de la tolerancia a la glucosa ${ }^{16}$.

$\mathrm{Al}$ analizar la variable grasa corporal, respecto a la duración del sueño, los resultados sugieren que los individuos calificados con porcentaje de grasa normal, presentan el porcentaje más bajo de cumplimiento durante la semana, sin embargo, esta diferencia no es estadísticamente significativa, lo cual no coincide con lo encontrado por Ruiz et a ${ }^{16}$, quienes evaluaron a 89 escolares en Venezuela, encontrándose que el sueño reducido está asociado a exceso de peso.

Otro estudio realizado en Quebec a 550 niños con una edad promedio de 9,6 años reveló la asociación de la duración del sueño con la adiposidad, la cual es independiente de la actividad física e ingesta calórica que pudiesen mediar esta relación ${ }^{17}$. Además, se debe considerar los resultados reportados el año 2015, en un estudio realizado a 66.817 adolescentes chinos que reveló asociaciones positivas entre sobrepeso y obesidad y horas de sueño ${ }^{18}$.

Una debilidad del estudio, es que al ser este de corte transversal, se dificulta la determinación de causalidad.

Las fortalezas se encuentran en la utilización de una encuesta de sueño validada internacionalmente y en la incorporación de la variable grasa corporal dentro de las mediciones antropométricas. Por otra parte, el estudio fue aplicado a habitantes de una región extrema, la cual, por sus características geográficas, presenta días muy largos o cortos, factor que podría influenciar en la duración de horas las de sueño de sus pobladores, generando la necesidad de continuar con la línea de investigación.

\section{CONCLUSIONES}

La malnutrición por exceso, constituye el principal problema de salud pública de la región de Magallanes. Los resultados aportados por la investigación, sugieren que a medida que aumenta el peso corporal en los escolares, existe una tendencia a disminuir las horas de sueño, siendo este un factor de riesgo para la malnutrición por exceso, por lo tanto, es de suma importancia continuar investigando la variable sueño en la población escolar magallánica y evidenciar la necesidad de incorporar este factor en los diversos programas de prevención y promoción de la salud a nivel escolar.

\section{RESUMEN}

El objetivo del estudio fue conocer el estado nutricional y la duración habitual de sueño en días de semana y fin de semana de escolares entre 6 a 15 años de la ciudad de Punta Arenas. Sujetos y métodos: Se obtuvo una muestra aleatoria simple de 481 escolares de 2 establecimientos educacionales (uno particular-subvencionado y uno municipalizado). Para diagnosticar el estado nutricional, se evaluó peso, talla y pliegue cutáneo tricipital. Para conocer la duración habitual del sueño se aplicó la versión española del Pediatric Sleep Questionnaire y los resultados se compararon con la recomendación entregada por la National Sleep Foundation. Resultados: El 60,4\% de los escolares presentó malnutrición por exceso, durmiendo en promedio $8,70 \pm 1,07$ horas los días de semana y $9,85 \pm 1,55$ horas los fines de semana. Conclusión: Los escolares diagnosticados con obesidad fueron los que presentan un menor cumplimiento de la recomendación, sin embargo, no hubo diferencia significativa entre ambas variables.

Palabras clave: Obesidad, escolares, estado nutricional, horas de sueño.

\section{BILBIOGRAFÍA}

1. Service light and obscurity. Government of Chile. Hydrographic and oceanographic service of the navy; 2017.

2. Report Nutritional Map. Government of Chile. National Board of Scholarships and Scholarships; 2015.

3. Muqing Cao, Yanna Zhu, Baoting He, Wenhan Yang, Yajun Chen, Jun Ma, Jin Jing. Association between sleep duration and obesity is age- and gender-dependent in Chinese urban children aged 6-18 years: a cross-sectional study. BMC Public Health. 2015;15:1029. doi: 10.1186/s12889-015-2359-0.

4. Fonken L, Nelson J. The effects of light at night on circadian clocks and metabolism, Endocr Rev 2014; 35(4): 648-670.

5. Bueno A, Olavarrieta S. Bernardino.F, Fernández Mendoza. Sleep and stress: relationship with obesity and metabolic síndrome. Rev Esp Obes 2007; 5(2): 77-90.

6. Masalán A, Sequeida Y, Ortiz M. Sleep and schoolchildren and adolescents, their importance and promotion through educational programs: Education and behavioral approach programs, Rev Chil Ped 2012; 84(5): 554-564.

7. Technical Norm of Nutritional Evaluation of the child from 6 to 18 years. Government of Chile. Ministry of Health, Government of Chile; 2003.

8. Barrera G. Nutritional Evaluation of Growth and Cardiovascular and Metabolic Risk. Institute of Nutrition and Food technology. University of Chile 2015, p.200-207.

9. Hirshkowitz, M et al. National Sleep Foundation's updated sleep duration recommendations: final report. Sleep Health: I National Sleep Found 1(4): 233-243.

10. Huamani C, Rey de Castro J. Drowsiness and sleep characteristics 
in schoolchildren in an urban district of Lima, Peru. Argentine archives of pediatrics 2014; 112(3): 239-241.

11. Calamaro C, Mason T, Ratcliffe S. Adolescents Living the 24/7 Lifestyle: Effects of Caffeine and Technology on Sleep Duration and Daytime Functioning. Pediatrics 2009; 123: 5-10.

12. Durán $S$, Fuentes de la $C N$, Vásquez $S$, Cediel $G$, Diaz V. Relationship between nutritional state and dream in schools of the commune of San Miguel, Santiago, Chile. Rev Chil Nutr 2012; 39(1): 30-37.

13. Durán S, Haro P. Association between the amount of sleep and obesity in Chilean Schoolchildren. Arch Argent Pediatr. 2016; 114(2): 114-119.

14. Carillo-Larco RM, Bernabé- Ortiz A. Miranda JJ. Short Sleep duration and childhood obesity: cross-sectional analysis in Perú and paterns in four developing countries. Plos One 2014; 9(11): e112433.

15. Knutson KL, Spiege/ K, Penev Pet al. The metabolic consequences of sleep deprivation. Sleep Med Rev 2007; 11: 163-178.

16. Ruiz, N, Rangel, A, Rodríguez C, Rodríguez L, Rodríguez V. Relación entre el déficit de sueño nocturno, el exceso de peso y las alteraciones metabólicas en adolescentes. Arch Argentinos Ped 2014; 112(6): 511-518.

17. Chaput JP, Lambert M, Gray-Donald K, McGrath IJ, Tremblay MS, O'Loughlin J, Tremblay A. Short sleep duration is independently associated with overweight and obesity in Quebec children. I Public Health 2011; 102(5): 369-374.

18. Wu J, Wu H, Wang J, Guo L, Deng X, Lu C. Asociations between Sleep Duration and Overweight/Obesity: Results from 66,817 Chinese Adolescents. Sci Rep 2015; 16; 5: 16686. Doi: 10.1038/ srep16686. 